08

\title{
Оптический аналог зонной плавки при комнатной температуре
}

\author{
(C) В.Н. Стрекалов \\ Московский государственный технологический университет „СТАНКИН“, \\ 127994 Москва, Россия \\ e-mail: vns1024@yandex.ru
}

Поступило в Редакцию 11 июля 2018 г.

В окончательной редакции 1 декабря 2018 г.

Принято к публикации 1 декабря 2018 г.

\begin{abstract}
Найдены условия, при которых рождение фотоэлектронов приводит к появлению в полупроводнике или прозрачном диэлектрике силы, вызывающей дрейф примесей. Дрейф происходит в направлении смещения фокальной области излучения, возбуждающего неосновные носители заряда. Использование этого условия позволило показать, что дрейф может быть более заметен, чем диффузия. Процесс можно считать оптическим аналогом зонной плавки, особенно важным в тонких пленках.
\end{abstract}

DOI: $10.21883 / J T F .2019 .05 .47479 .268-18$

Основная цель настоящей работы - дать описание механизма неравновесного рассеяния неосновных носителей (фотоэлектронов), приводящего к возбуждению в конденсированных средах оптического эффекта, аналогичного зонной плавке. В отличие от стандартной модели зонной плавки этот механизм не требует нагрева вещества выше температуры плавления, и потому возможен в тонких пленках без ее проплавления.

В физике конденсированных сред придают большое значение изучению свойств дефектов и примесей, в том числе динамике последних под воздействием различных внешних факторов [1-3]. Определенное внимание уделяется усилиям по тонкой (на атомном уровне) обработке поверхностей [4-6], а также управлению механическим движением примесей внутри образца. Классическим примером такого управления является зонная плавка. Не менее интересны эффекты восходящей диффузии и сегрегации примесей, в которых возникает направленное движение примесей в механически деформированной решетке или при облучении нейтронами, протонами или ионами $[7,8]$.

Движение заряженных примесей в образцах, помещенных в электрическое поле, носит известный диффузионно-дрейфовый характер $[9,10]$.

Имеется и другие похожие процессы управляемого перемещения примесей. Это неоднородный нагрев вещества лазерным излучением или мощным некогерентным источником, а также прямое поглощение связанными в решетке примесями (как в работах [11-13]). Частично квантовокинетическая задача о неравновесной диффузии была решена в модели усеченного гармонического осциллятора $[12,13]$. В этой модели квант $\hbar \omega$, поглощенный гармоническим осциллятором (примесью в решетке), приводит к подъему частицы в потенциальной яме, в которой она связана. В результате эффективный потенциальный барьер, который необходимо преодолеть при диффузии или десорбции примеси, уменьшается от величины $U_{0}$ до величины $U_{0}-\hbar \omega$, а это увеличивает аррениусовскую экспоненту $\exp \left\{-U_{0} / T\right\}$ до значения $\exp \left\{-\left(U_{0}-\hbar \omega\right) / T\right\}$, где $T-$ температура системы в энергетических единицах. Изменение аррениусовской экспоненты приводит к резкому увеличению скорости диффузии, доводя ее при разумных параметрах до скорости диффузии в жидкости. При этом реального плавления или нарушения дальнего порядка решетки не происходит [11-13].

Вопросы управления движением примесей лазерным излучением рассмотрены и в работе [14]. Эта работа относится только к сегнетоэлектрикам и основана на представлении о дрейфе носителей и примесей в постоянном поле самопроизвольной поляризации сегнетоэлектрика при дополнительном нагреве образца.

Рассмотрим другой процесс управляемого перемещения примесей. Механизм этого процесса - возникновение механических сил (давления или механических напряжений), действующих на примесим со стороны родившихся неосновных носителей с большими, по сравнению с тепловыми, импульсами. Неоднородный нагрев вещества лазерным излучением или мощным некогерентным источником, а также прямое поглощение связанными примесями - гармоническими осцилляторами (как в работах [12,13]) не будут учитываться, хотя достаточно очевидно, что эти процессы могут быть важны.

Пусть имеется примесь в кристаллической потенциальной яме глубиной $U_{0}$. В равновесных условиях для совершения диффузионного скачка в одну из соседних ячеек, энергия примеси должна испытать тепловую флуктуацию с энергией, близкой к $U_{0}$. Другая возможность скачка - это совершение над примесью работы внешней силы $\mathbf{F}$

$$
A=\mathbf{a F} \geq U_{0}
$$

где $\mathbf{a}$ - вектор скачка, при котором внешняя сила совершает работу над примесью; $|\mathbf{a}|$ имеет порядок одной или нескольких постоянных кристаллической решетки. 
Скалярное произведение в (1) учитывает анизотропию процессов диффузии и дрейфа, которая обеспечивает нулевой вклад в процесс в направлениях, перпендикулярных направлению действия силы.

Природа силы F, вообще говоря, произвольна. Здесь мы рассмотрим силу, с которой на примесь действует давление неосновных носителей заряда, созданных лазерным излучением или некогерентным источником достаточной мощности при ионизации примесей или внутреннем фотоэффекте.

Очевидно, что однородное или изотропное распределение носителей не будет вызывать направленного потока частиц (хотя в этом случае нагрев носителей или вещества образца ускорит диффузию). Будем предполагать, что образец освещается цилиндрической линзой, длина которой порядка ширины образца. Линза создает гауссовское распределение интенсивности с полушириной $L_{0}$ порядка длины волны излучения. Сканирование фокальным пятном обычной линзы при постоянном или скачкообразном перемещении пятна поперек направления основного движения также приведет к ожидаемому эффекту. Однако в этом случае будут возникать непродуктивные потери энергии излучения на дополнительный нагрев вещества и работу по перемещению примеси „туда-сюда“в поперечном направлении движения пятна. Поэтому выбор цилиндрической линзы предпочтительнее.

В случае неподвижной линзы возможно локальное перераспределение примесей - уход примесей из центральной области с наибольшей интенсивностью излучения и наибольшей концентрацией рожденных неосновных носителей. Движущаяся освещенная область несколько напоминает передний фронт зонной плавки. При этом следует учесть, что процессы рождения и нагрева носителей на переднем фронте, как правило, имеют намного большую скорость, чем релаксационные процессы на заднем фронте. Это означает, что градиенты параметров носителей „впереди“ (в дальнейшем область 1) больше, чем „позади“ (область 2) по отношению к максимуму освещенности. Соответственно различаются действующие градиентные силы.

Кроме того, как и в зонной плавке, концентрация примесей в области заднего фронта меньше, чем концентрации в области переднего фронта (процесс очистки образца). Поэтому процессы в области 2 не будут рассматриваться.

При указанных условиях возникнет диффузионнодрейфовое движение примесей в направлении движения фокальной области, обсуждение которого является целью настоящей работы. По-видимому, процесс диффузионно-дрейфового движения в световом поле наиболее интересен и эффективен в случае глубоких примесей, когда тепловые возбуждения переходов уровень - разрешенная зона играют незначительную роль.

Рассмотрим электромагнитное излучение, энергия фотона которого удовлетворяет неравенствам (внутренний фотоэффект пока не учитываем)

$$
E_{A}, E_{D}<\hbar \omega<E_{G}
$$

где выделены энергии активации акцепторов или доноров и ширина запрещенной зоны. Это позволит рассматривать только переходы валентная зона--акцепторный уровень или переходы донор-зона проводимости, т.е. режим относительно слабого линейного поглощения света.

Учитывая коэффициент отражения $R_{0}$ и линейный коэффициент поглощения $\alpha$, находим, что интенсивность излучения убывает с глубиной $Z$ по закону Бугера:

$$
J(x, y, z)=J_{0}(1-R) \exp \{-\alpha Z\} \exp \left\{-\frac{\left(X-X_{0}\right)^{2}}{L_{0}^{2}}\right\},
$$

причем $X_{0}=V_{0} t$, где $V_{0}-$ скорость движения фокуса линзы. Зависимости от $Y$ нет, так как используется цилиндрическая линза.

Вводя в рассмотрение интенсивность излучения, легко заметить, что поглощенная в единице объема в единицу времени энергия равняется

$$
P(X, Z)=\alpha J(X, Y, Z) .
$$

Если квантовый выход рождения неосновных носителей близок к единице, то скорость рождения носителей равна скорости поглощения фотонов, т.е.

$$
\frac{d N}{d V d t}=\frac{\alpha}{\hbar \omega} J(X, Y, Z),
$$

где $N$ - число родившихся неосновных носителей.

Учтем теперь, что каждый неосновной носитель рождается с импульсом

$$
P_{0}=\sqrt{2 m\left(\hbar-E_{D}\right)} .
$$

После рождения носителя его импульс релаксирует из-за столкновений с другими квазичастицами, однако благодаря малым потерям энергии (например, при столкновениях с фононами), импульс (6) сохраняется с малыми изменениями ,достаточно долго“. Это приближения подтверждается при квантовокинетическом рассмотрении процесса.

Формула (6) записана для случая донора (формула для акцептора аналогична (6), но отличается эффективной массой носителя и энергией активации).

Заметим теперь, что к дрейфу приводят не все неравновесные носители.

Во-первых, в любом заданном направлении движется только $1 / 6$ носителей.

Во-вторых, частицы, движущиеся вдоль оси $Y$, не вызывают дрейфового движения в силу симметрии системы.

B-третьих, носители заряда, движущиеся вдоль оси $Z$, „загоняют“ примеси в глубину образца. В настоящей работе мы не будем обсуждать этот эффект. 
Далее надо учитывать, что „нужные“ носители заряда рассеиваются на всех примесях, число которых можно принять равным $n(X)=N(X)$, т. е. ионизованных светом (сечение рассеяния на нейтральных примесях, как известно, меньше).

Наконец, различна роль носителей, движущихся в областях 1 и 2 вдоль оси $X$. Вблизи переднего фронта концентрация частиц и градиенты больше (в области 2 носители родились раньше и успели частично релаксировать по энергии или рекомбинировать). Кроме того, в области 2 концентрация примесей меньше изза их частичного дрейфа в первую область. Поэтому (повторим еще раз) будет учитываться только движение носителей заряда из фокальной области в область 1 .

Взаимодействовать с конкретной примесью могут только носители, находящиеся в цилиндре с основанием $\sigma$ (площадь поперечного сечения рассеяния, например, круг с радиусом дебаевской экранировки) и высотой $L$, физический смысл которой обсуждается ниже.

В результате число „активных“ носителей равно

$$
N(X)=\frac{\alpha \sigma L}{6 \hbar \omega} J(X, Y, Z) .
$$

Если учесть сказанное (формулы (3), (5) и (7)), то можно записать силу, действующую на примесь:

$$
\begin{gathered}
F=\frac{\sigma L}{6} \frac{\alpha J_{0}}{\hbar \omega} P_{0}\left(1-R_{0}\right) \exp \{-\alpha Z\} \exp \left\{-\frac{\left(X-X_{0}\right)^{2}}{L_{0}^{2}}\right\}, \\
\mathbf{F}=\mathbf{i} F
\end{gathered}
$$

Условием возникновения обсуждаемого эффекта будет неравенство (1), которое с учетом результата (8) принимает вид

$$
\begin{aligned}
a F= & \frac{\sigma L}{6} a \frac{\alpha J_{0}}{\hbar \omega} P_{0}(1-R) \exp \{-\alpha Z\} \\
& \times \exp \left\{-\frac{\left(X-X_{0}\right)^{2}}{L_{0}^{2}}\right\} \geq U_{0}
\end{aligned}
$$

Важно подчеркнуть, что силу $F$ будет определять именно импульс неосновного носителя, как импульс, передаваемый примеси при рассеянии на ней носителя (по понятной причине получаем несколько заниженное значение силы).

Под величиной $L$ следует понимать минимальное расстояние, выбранное из величин:

- длины экранировки (в случае заряженной примеси),

- длины свободного пробега неравновесного носителя до столкновения с примесью,

- кубического корня из обратной концентрации родившихся носителей,

- расстояния между донорными (или акцепторными) примесями.

Набор возможных длин $L$, а также значительное число параметров, входящих в пороговое условие (9), означает, что проводить реальные оценки целесообразно только для конкретной экспериментальной картины.

Возникает вопрос, будет ли дрейфовое движение превосходить диффузионное расплывание. Для ответа на этот вопрос учтем, что поток числа диффундирующих и дрейфующих частиц определяется соотношением $[9,10]$ для потока частиц

$$
J(X)=V n(X)-D \frac{d n(X)}{d X}=\mu n(X) F(X)-D \frac{d n(X)}{d X},
$$

где $\mu$ и $D-$ подвижность примесей и коэффициент их диффузии.

Используя соотношение Эйнштейна-Смолуховского $D=\mu T$ и уравнение (10), можно записать неравенство, выполнение которого означает, что основным процессом переноса примесей будет дрейф,

$$
F(X)>\left|\frac{1}{n(X)} \frac{d n(X)}{d X}\right| .
$$

Для реального использования условия (11) необходимо найти точки $X_{0}$, в которых достигается максимум силы, что возможно только для конкретной схемы эксперимента.

Работа выполнена при поддержке Министерства образования и науки РФ (гос. задание № 9.1195.2017/4.6).

\section{Список литературы}

[1] Вавилов В.Е., Кив А.Е., Ниязова О.Р. Механизмы образования и миграции дефектов в полупроводниках. М.: Наука, 1981.

[2] Косевич А.М. Физическая механика реальных кристаллов. Киев: Наук. думка, 1981.

[3] Гегузин Я.Е. //УФН. 1986. Т. 149. Вып. 1. С. 149-159.

[4] Простнев А.С., Шуб Б.Р. // Химическая физика. 2012. T. 31. № 1. C. $72-78$.

[5] Стрекалов В.Н. // Письма в ЖТФ. 1990. Т. 16. Вып. 21. C. $84-88$.

[6] Стрекалов В.Н. // ФТП. 1986. Т. 20. Вып. 10. C. $1939-1942$.

[7] Зон Б.А., Ледовский С.Б., Лихолет А.Н. // ЖТФ. 2000. Т. 70. Вып. 4. С. $38-41$.

[8] Фельдман Э.П., Юрченко В.М. // Поверхность. Физика. Химия. Механика. 1990. Т. 12. С. 138-147.

[9] Зельдович Я.Б., Мышкис А.Д. Элементы математической физики. М.: Наука, 1973.

[10] Левич В.Г. Курс теоретической физики. Т. 1. М.: ФМ, 1962.

[11] Стрекалов В.Н. // ФТП. 1986. Т. 20. Вып. 2. С. 361-363.

[12] Strekalov V.N., Strekalov D.V. // Phys. Rev. A. 2001. Vol. 63. P. 032901.

[13] Strekalov V.N., Strekalov D.V. // Phys. Rev. A. 2002. Vol. 65. P. 062901.

[14] Sturman B., Kosters M., Haertle D., Becher C., Buse K. // Phys. Rev. B. 2009. Vol. 80. C. 245319. 